\title{
SLE presenting as DAH and relapsing as refractory retinitis
}

\author{
Somya Ish, ${ }^{1}$ Deepa Sharma, ${ }^{1}$ Pranav Ish ${ }^{2}$ \\ ${ }^{1}$ Department of Ophthalmology, Dr RML hospital; ${ }^{2}$ Department of Pulmonary, Critical Care and Sleep Medicine, \\ Safdarjung Hospital, New Delhi, India
}

Dear Editor,

A 17-year-old boy, diagnosed with Systemic Lupus Erythematosus (SLE), presented to ophthalmology department with gradual painless diminution of vision in both eyes (right more than left). He had already received 6 pulses of cyclophosphamide and steroids at monthly intervals one year back for diffuse alveolar hemorrhage (DAH)[1] and was on maintenance oral $40 \mathrm{mg}$ prednisolone and 3 grams mycophenolate mofetil (MMF). There was no history of oliguria, skin rash, joint pain, oral ulcers, photosensitivity or any neurological deficit in this presentation. There was no proteinuria, hematuria or worsening of renal function. The Serum Antinuclear Antibody (ANA) and dsDNA were

Correspondence: Pranav Ish, Department of Pulmonary, Critical Care and Sleep Medicine, Safdarjung Hospital, New Delhi 110029.

Tel.: 9958356000.

E-mail: pranavish2512@gmail.com

Key words: Retinitis; Rare; Systemic Lupus Erythematosus; DAH.

Funding: None.

Conflict of interest: The Authors declare no conflict of interest.

Availability of data and materials: All data underlying the findings are fully available.

Ethics approval and consent to participate: No ethical committee approval was required for this case report by the Department, because this article does not contain any studies with human participants or animals. Informed consent was obtained from the patient included in this study.

Consent for publication: The patient gave his written consent to use his personal data for the publication of this case report and any accompanying images.

Contributions: All the authors contributed equally. All the authors have read and approved the final version of the manuscript and agreed to be accountable for all aspects of the work.

Received for publication: 16 February 2020.

Accepted for publication: 18 June 2020.

${ }^{\circ}$ Copyright: the Author(s), 2020

Licensee PAGEPress, Italy

Monaldi Archives for Chest Disease 2020; 90:1250

doi: 10.4081/monaldi.2020.1250

This article is distributed under the terms of the Creative Commons Attribution Noncommercial License (by-nc 4.0) which permits any noncommercial use, distribution, and reproduction in any medium, provided the original author(s) and source are credited. positive along with low serum complement levels. There was no dyspnea, oxygen saturation was preserved (99\%) and chest X-Ray performed was normal. The rare diagnosis of SLE without renal involvement with systemic manifestations including DAH earlier and ocular flare in the present admission was kept.

On ocular examination, best corrected visual acuity right eye (RE) was 6/60 and left eye (LE) was 6/36. The anterior segment examination was normal in both eyes. On fundus examination of right eye, media was hazy due to mild vitritis. There were coalescent hypopigmented lesions with fluffy margins superotemporal to disc, suggestive of active retinitis, along with macular edema (Figure 1a) in the RE with corresponding frank leakage on Fundus fluorescein angiography (FFA). (Figure 1b) In the
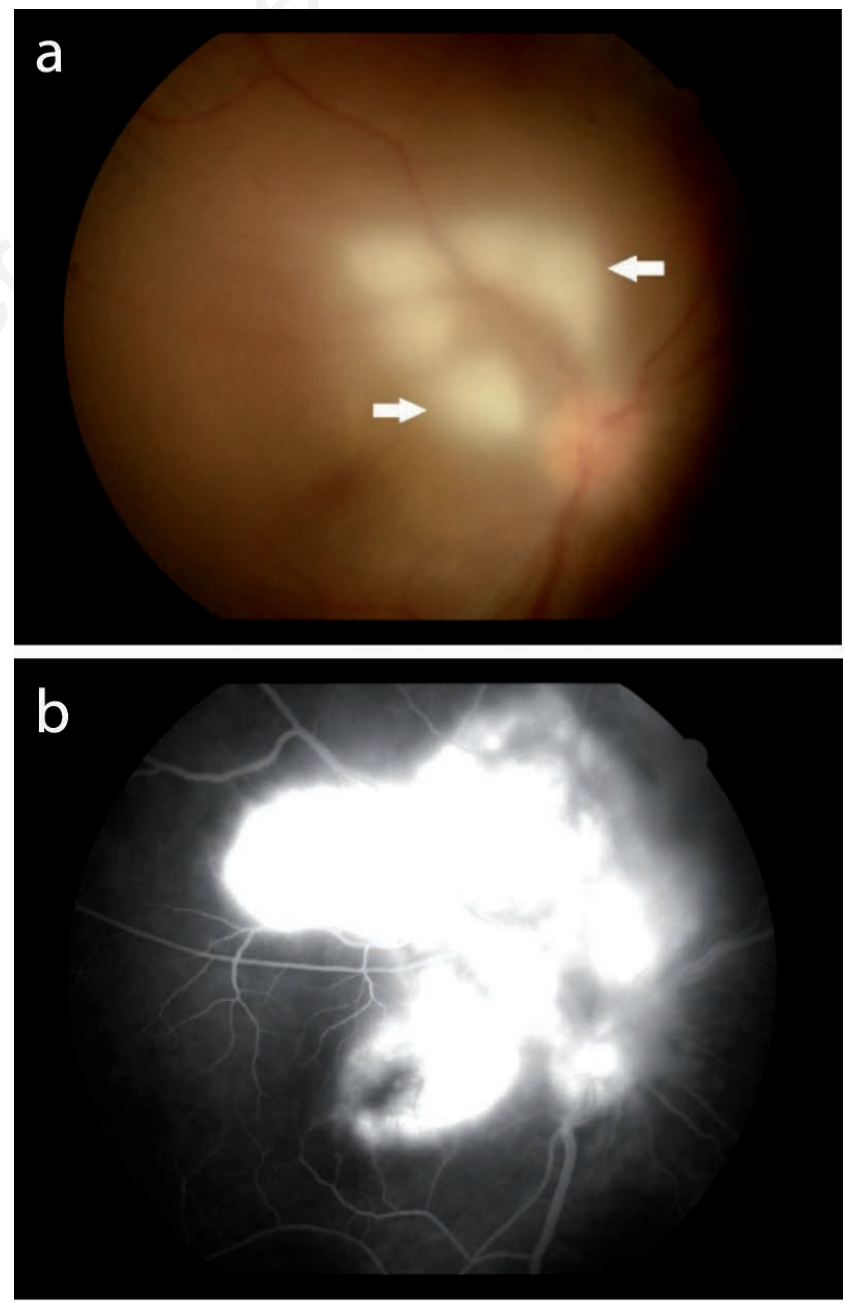

Figure 1. a) Right eye active retinitis lesions superotemporal to disc (white arrows). b) Frank leakage on FFA in Right eye corresponding to active retinitis lesions with macular edema. 
LE, there were similar lesions in inferotemporal quadrant with macular edema (Figure 2a), which also showed frank leak on FFA (Figure 2b).

In view of favorable response in the past, the patient was given three pulses of cyclophosphamide (750 mg for 1 day) along with methylprednisolone ( 1 gram for 3 days) at monthly interval; but there was no response and further deterioration in vision of $\mathrm{RE}$ (finger counting present at distance of 4 meters).

The patient was then shifted to rituximab $500 \mathrm{mg}$ weekly pulse for 4 doses and continued on MMF. In RE, best corrected visual acuity (BCVA) was maintained at finger counting at 4 meters. The initial retinitis lesions healed but a gliotic band was formed superior to disc extending to inferotemporal arcade through the fovea and new retinitis lesions were present superotemporal and inferotemporal to fovea (Figure 3a). In LE, BCVA improved to 6/24 with healing of retinitis lesions with scarring (Figure $3 b$ ) thereby leading to clinical recovery. The patient was shifted back to oral prednisolone at increased dose of $60 \mathrm{mg}$ and continued on 3 grams mycophenolate mofetil on discharge.
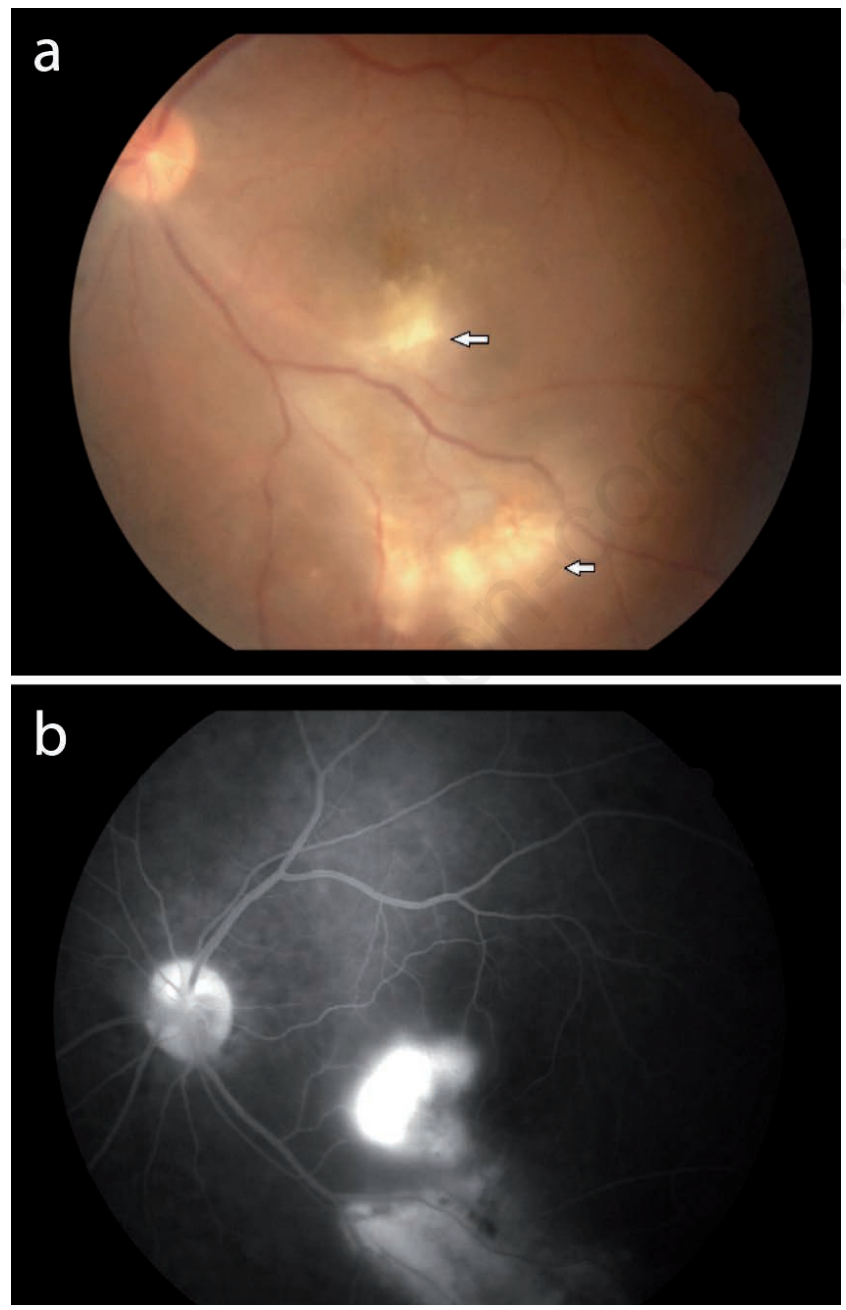

Figure 2. a) Left eye active retinitis lesions inferior to fovea and in inferotemporal quadrant (white arrows). b) Frank leakage on FFA in Left eye corresponding to active retinitis lesions with macular edema.
Retinal vasculitis is uncommon in SLE, affecting only $3 \%$ to $11 \%$ of lupus patients [2]. Occasionally, it may be sight threatening requiring aggressive therapy. Rituximab has been successfully used in isolated cases of refractory disease and large-scale studies are needed to provide evidence for guidelines formulation for treatment of such cases $[3,4]$.

The pathogenesis of lupus retinitis remains unclear, but a central role of B cells, antibodies, immune-complex formation and complement activation has been postulated [5], which is further justified by the partial yet clinically significant response to rituximab as seen in this case.

To conclude, a patient with SLE retinitis with no renal or other typical organ involvement is uncommon but can be sight threatening. The patient in this scenario had a background diagnosis of SLE which itself had an uncommon presentation as DAH. This case highlights the systemic nature of SLE and the need to keep a regular follow up along with a high index of suspicion for rare systemic manifestations of SLE, to decrease the morbidity and mortality.
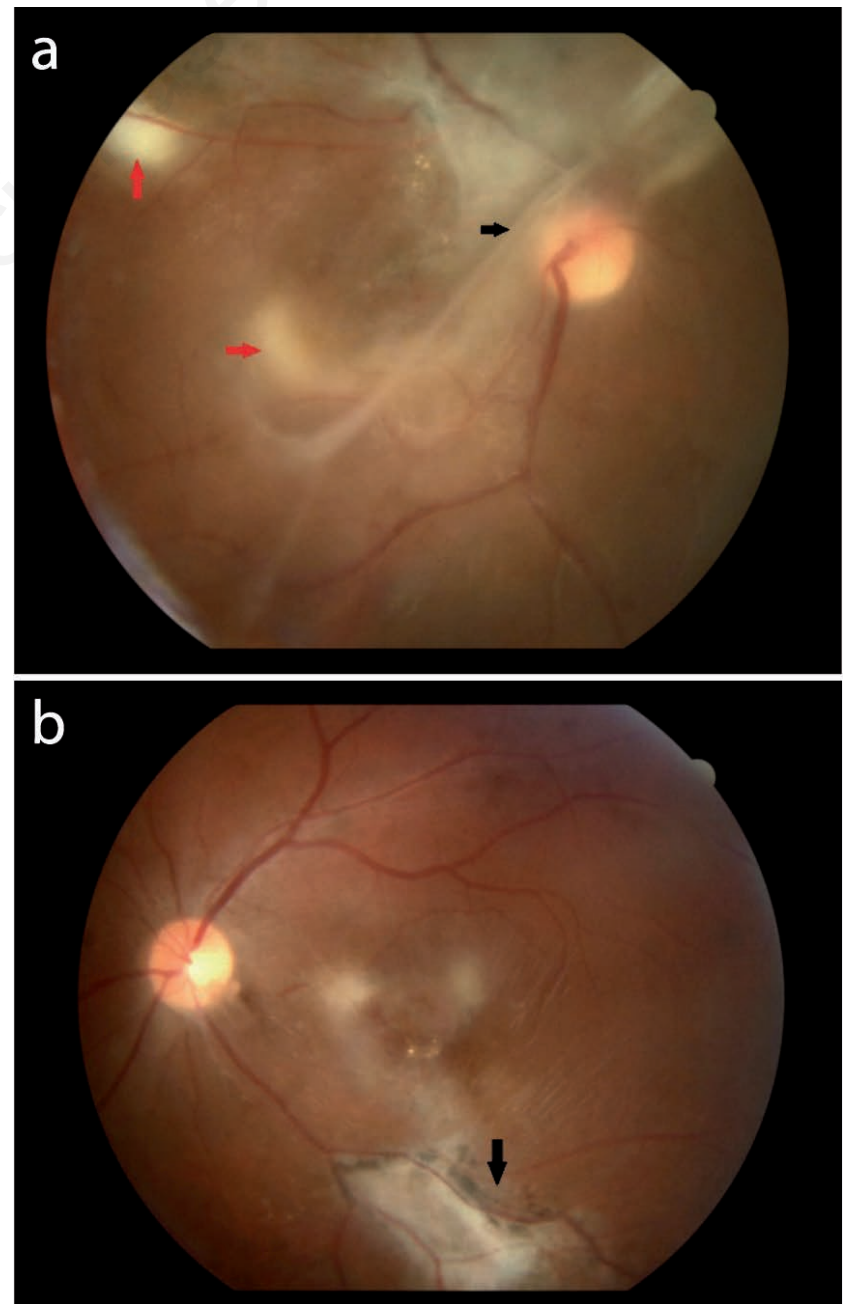

Figure 3. a) Gliotic band (black arrow) with new retinitis lesions (red arrow) in right eye. b) Healed lesions with scarring in left eye (black arrow). 
3. Donnithorne KJ, Read RW, Lowe R, et al. Retinal vasculitis in two pediatric patients with systemic lupus erythematosus: a case report. Pediatr Rheumatol Online J 2013;11:25.

1. Mittal A, Suri JC, Chakrabarti S, Ish P. An uncommon cause of diffuse alveolar hemorrhage in a young male. Monaldi Arch Chest Dis. 2019;89.

2. Tselios K, Lam WC, Urowitz MB, Gladman DD. Rituximab for Sight-Threatening Lupus-Related Retinal Vasculitis. J Clin Rheumatol 2018;24:93-4

4. Hickman RA, Denniston AK, Yee CS, et al. Bilateral retinal vasculitis in a patient with systemic lupus erythematosus and its remission with rituximab therapy. Lupus 2010;19:327-329.

5. Eisenberg R. Mechanisms of autoimmunity. Immunology Res 2003;27:203-218. 